\title{
SHORT COMMUNICATION Time Jitter in Phase Controlled Thyristor Circuits
}

\author{
J. K. HALL and D. S. PALMER \\ Dept. of Electronic and Electrical Engineering, University of Technology, Loughborough, Leicestershire
}

(Received July 7, 1975)

\section{INTRODUCTION}

It is frequently assumed that radio interference generated by thyristor circuits is entirely due to the harmonics of the output waveform. However a closer examination of the higher frequency spectrum of typical phase controlled circuits reveals that the spaces between the harmonic lines are infilled with what appears to be noise. ${ }^{1}$ This communication presents some of the early findings of an investigation into this phenomenon.

\section{POSSIBLE SOURCES OF NOISE}

Three sources of noise may be postulated at the outset, the thyristor, the firing system and the mains supply. Noise may also be produced by interaction between these sources in some cases. Of particular importance in this respect is the time jitter in the period of conduction of the thyristor, and between the conducting periods. This jitter is caused by random variations in the time the thyristor commences and ceases conduction.

The possible types of noise produced in semiconductor devices are well documented. ${ }^{2}$ In so far as time jitter due to the thyristor turn-on process is concerned, only random effects in the avalanche can cause variation in turn-on time from cycle to cycle if all the external parameters of the thyristor such as gate voltage and current, and its rate of rise, anode voltage and junction temperature are held constant. Any variations in timing of the gate signal provided by the firing circuit will produce jitter in the output waveform.

Two variable properties of the mains supply may influence timing jitter. Changes in load flow in the supply system impedance cause fluctuations both in the timing of the zero crossings about a mean position, and in the amplitude of the voltage. If the zero crossings of the supply voltage are not fixed at precisely regular intervals in time, neither can be the turn-on nor the turn-off of the thyristor. In most simple firing circuits the time delay is produced by charging a capacitor from the mains through a resistor. Fluctuation of the mains amplitude will therefore cause further turn-on timing errors.

\section{EFFECTS OF TIME JITTER}

The precise effects of time jitter upon the output of a phase controlled thyristor circuit and upon the supply current drawn by that circuit will depend upon its source. If all the jitter present is introduced by the firing circuit, then only the steep front edge of the waveform will move in time, the rest of the waveform being stationary. On the other hand if all the jitter is produced by mains irregularities then both leading and trailing edges of the waveform will move randomly in time.

In the latter case the resultant waveform may be expressed mathematically as:

$$
F(t-D q(T))=\sum_{n=0}^{\infty} A_{n} \cos \frac{2 \pi n}{T}(t-D q(t))^{3}
$$

where $F(t)$ is the unjittered time waveform

$D$ is a constant with the dimensions of time

$q(t)$ is a dimensionless function of time

so that $F(t-D q(t))$ is the time jittered waveform

$A_{n}$ is the peak amplitude of the $n$th harmonic

$T$ is the fundamental period of the waveform $t$ is time.

The $n$th harmonic may be written as

$$
F_{n}(t-D q(t))=A_{n} \cos \frac{2 \pi n}{T}(t-D q(t))
$$


A phase modulated carrier wave may be written as

$$
y(t)=C \cos \left(\frac{2 \pi t}{T_{c}}+m_{p} q(t)\right)
$$

Where $C$ is the peak carrier amplitude

$T_{\mathrm{c}}$ is the period of the carrier

$m_{p}$ is the phase modulation index.

Comparing Eq's (2) and (3) it can be seen that, if the period of the $n$th harmonic $T / n$ is substituted for the carrier period $T_{c}$ and if $2 \pi n D / T$ is substituted for $m_{p}$, the two equations are identical in form. This demonstrates that each harmonic of the jittered waveform is phase modulated. An important conclusion which may be drawn from this is that the phase modulation index $m_{p}=2 \pi n D / T$ is proportional to the harmonic number. Thus more pronounced phase modulation effects may be expected in the high frequency spectrum.

Consider the case where the fundamental waveform is jittered sinusoidally, the jittering frequency being appreciably lower than the fundamental frequency. The phase modulation produces a series of lines spaced by the jittering frequency on either side of the main spectral lines. The higher the main waveform harmonic, the more sideband lines will appear. If the jitter is random, the sideband lines are replaced by noise.

Jitter does not increase the total power contained in the spectrum; it merely redistributes it so that at higher frequencies the inter-line spaces become filled in with noise while the main lines fall in amplitude.

If the mains supply is fluctuating in amplitude, i.e. amplitude modulated, the effect on the spectrum will be that all the harmonics will be amplitude modulated with the same modulation index.

\section{RELATIVE IMPORTANCE OF THE JITTER NOISE SOURCES}

Experimental results obtained by comparing the spectra of thyristor output voltage waveforms when mains driven and when driven by a very stable inverter have demonstrated that: a) thyristor avalanche variation has little effect.

b) the firing circuit introduces only a small amount of noise when driven from a stable source; the "cheaper" circuits produce a higher noise level.

c) by far the worst source of jitter noise is the mains supply.

Confirmatory tests for (c) were made by severely clipping the mains and inverter voltages to produce virtually identical rectangular waveforms. No thyristor control was used. The interharmonic spaces in the clipped mains waveform spectrum showed high noise level, particularly at the higher frequencies, while the clipped inverter waveform did not. ${ }^{4}$

\section{CONCLUSIONS}

Most of the noise found between the higher harmonic lines is due to mains imperfections. Since there is little that the circuit designer can do about this, it is perhaps fortunate that when the usual steps are taken to remove the harmonic from the waveform the interharmonic noise will also be removed. The removal of the harmonics may be compared with the switching off of the carrier of a radio transmitter. If there is no carrier there can be no sidebands. If there are no harmonics there can be no noise due to jitter.

\section{ACKNOWLEDGEMENTS}

Acknowledgement is made of the experimental facilities provided by the Department of Electronic and Electrical Engineering, Loughborough University of Technology and to the Science Research Council for financial support. The advice and encouragement of Professor D. S. Campbell is greatly appreciated.

\section{REFERENCES}

1. J. K. Hall and N. C. Jones, Electrocom. Sci. and Tech., 1, 1, 43-49, (1974).

2. A. Van Der Ziel, Noise: Sources, Characterisation, Measurement, Prentice-Hall, (1970).

3. D. S. Palmer, R.F. Components Generated by Thyristor Gate Pulse Jitter, M.Sc. Project Report, Loughborough University of Technology, 1973.

4. D. S. Palmer and J. K. Hall, I.E.E. Conf. Publ. No. 123, 38-43, (December 1974). 

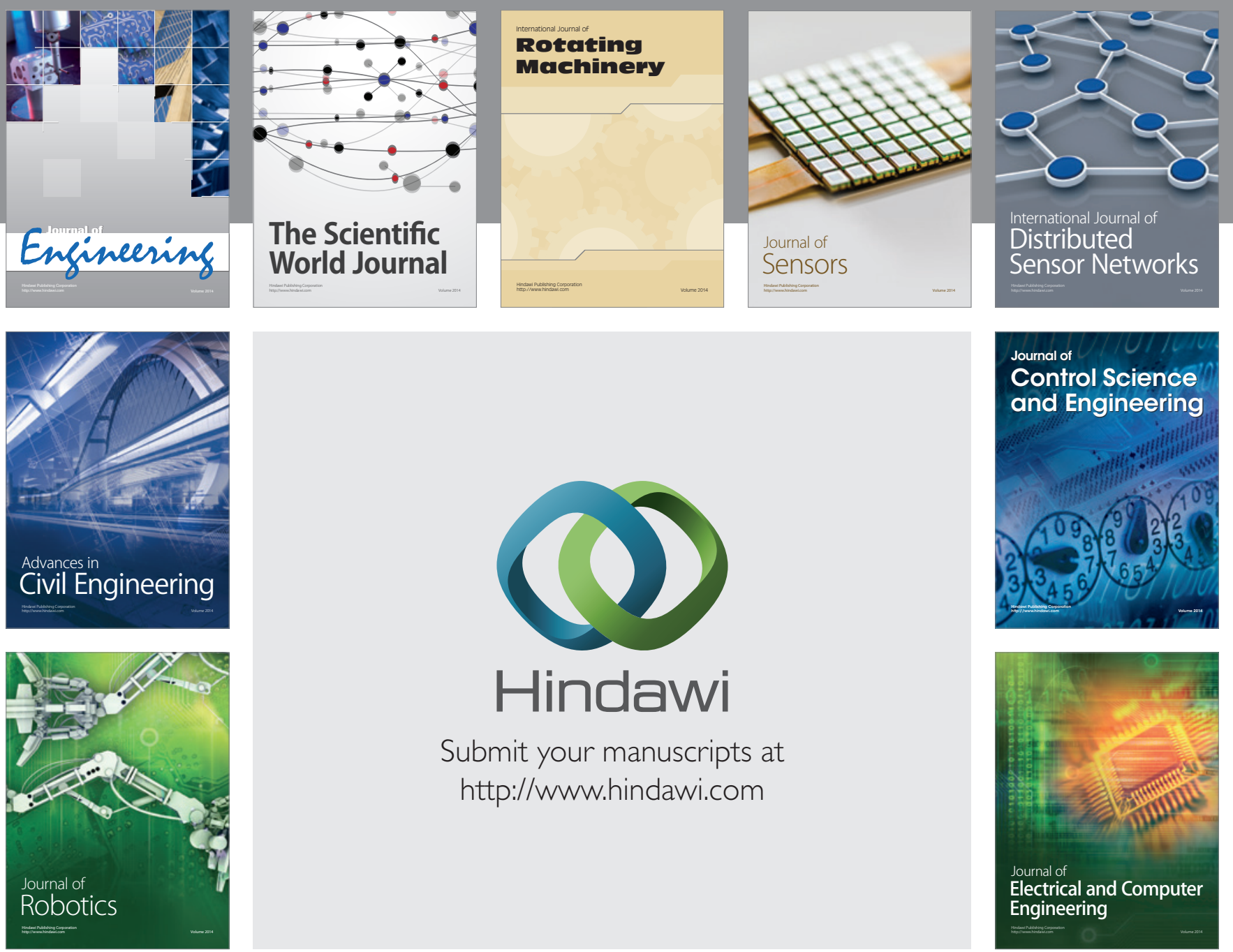

Submit your manuscripts at

http://www.hindawi.com
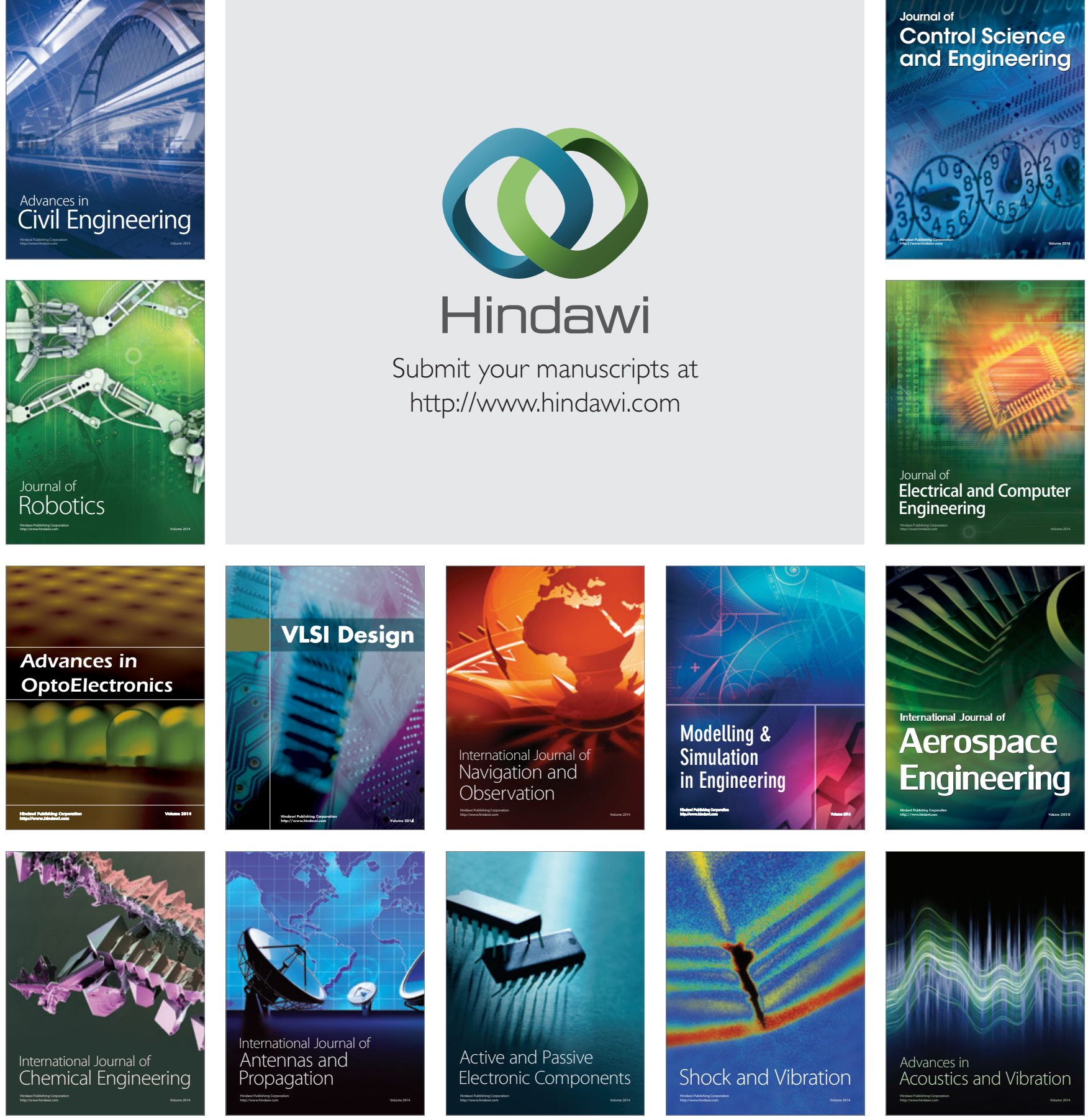